Los pequeños roedores de la Península de Yucatán:

conocimiento y perspectivas en 114 años de investigación

\title{
Small rodents in the Yucatan Peninsula: knowledge and perspectives in 114 years of research
}

\author{
Elisa P. Zaragoza-Quintana ${ }^{1}$, Juan M. Pech-Canché2, Javier E. Sosa-Escalante ${ }^{3}$, Silvia F. Hernández-Betancourt ${ }^{4}$, Livia S. \\ León-Paniagua ${ }^{5}$ and María C. MacSwiney G. ${ }^{1^{*}}$
}

\begin{abstract}
${ }^{1}$ Centro de Investigaciones Tropicales, Universidad Veracruzana. Ex-hacienda Lucas Martín s/n, Colonia Periodistas, 91090. Xalapa, Veracruz, México. E-mail: nasuanarik@yahoo.com.mx (EPZQ),cmacswiney@uv.mx (MCMG).

${ }^{2}$ Laboratorio de Vertebrados Terrestres, Facultad de Ciencias Biológicas y Agropecuarias, Universidad Veracruzana. Km. 7.5 Carretera Tuxpan-Tampico, 92850. Tuxpan, Veracruz, México. E-mail: jmpech@gmail.com (JMPC).

${ }^{3}$ Centro para la Gestión de la Sustentabilidad. Calle 78 No.578 entre 13-1 y 128, 97217. Mérida, Yucatán, México. E-mail: jeseara@ prodigy.net.mx (JESE).

${ }^{4}$ Departamento de Zoología, Facultad de Medicina Veterinaria y Zootecnia. Universidad Autónoma de Yucatán. Km 15.5 Carretera 97000, Mérida Xmatkuil. Mérida, Yucatán, México. E-mail: hbetanc@uady.mx (SFHB).

${ }^{5}$ Museo de Zoología, Departamento de Biología Evolutiva, Facultad de Ciencias, Universidad Nacional Autónoma de México. Circuito exterior s/n, Ciudad Universitaria, Copilco Coyoacán, 04510. Ciudad de México, México. E-mail: Ilp@ciencias.unam.mx (LSLP). ${ }^{*}$ Corresponding author
\end{abstract}

Studies conducted on small mammals, such as tropical rodents, have generated great interest since they can easily provide information concerning to their populations. The present article presents an analysis of the bibliography and sampling locations of small rodents of the Yucatán Peninsula in Mexico. In addition, the information contained within the literature is revised in order to identify the geographic and thematic coverage of the species records for the purpose of analyzing perspectives in the study of small rodents and in order to contribute to the efforts being made towards their protection and conservation. Publications of periodical journals, books and book chapters published between 1901 and 2015 were reviewed and analyzed. The literature covered research of the 15 small rodent species that are distributed on the Yucatán Peninsula. The studies were grouped into 10 themes, an accumulation curve was prepared by decade for each state of the Yucatan Peninsula and a map of distribution was generated detailing the locations of the records of each species. A total of 212 references were obtained, of which 162 corresponded to articles in periodical publications and 50 to books and book chapters. The most studied species were Peromyscus yucatanicus, Heteromys gaumeri and Ototylomys phyllotis; in contrast, those for which practically no information exists were Tylomys nudicaudus, Reithrodontomys spectabilis and Heteromys desmarestianus. The State of Yucatán has been the most explored and has the greatest number of sites and records of small rodents, followed by Quintana Roo and finally Campeche. The most commonly addressed themes were distribution, taxonomy, phylogeny, diseases and parasitism. Gaps in the information were detected for the majority of the species in the different themes such as feeding, reproduction, anatomy and morphology. For this reason, it is important to carry out studies in these themes in order to further the knowledge of the small rodents of the Yucatán Peninsula. These studies could provide information with the aim to propose changes to their conservation status in both Mexican and international legislation, or take appropriate decisions regarding protection of their specific habitats.

Los estudios realizados en pequeños mamíferos, como los roedores tropicales, han cobrado gran interés ya que proporcionan con cierta facilidad información acerca de sus poblaciones. En el presente artículo se realizó un análisis de la bibliografía publicada sobre los pequeños roedores de la Península de Yucatán, México. Asimismo, se realizó una revisión de la información contenida en la literatura para identificar la temática y cobertura geográfica de los registros de las especies, con el propósito de analizar las perspectivas en el estudio de pequeños roedores y contribuir a los esfuerzos encaminados a su protección y conservación. Se revisaron y analizaron publicaciones de revistas periódicas, libros y capítulos de libros realizados entre 1901 y 2015, sobre las investigaciones de las 15 especies de pequeños roedores que se distribuyen en la Península de Yucatán. Los trabajos se agruparon en 10 temas de investigación, se elaboró una curva de acumulación de especies por década para cada estado de la Península de Yucatán y se generó un mapa de distribución con las localidades de registro de cada especie. Se obtuvieron un total de 212 referencias, de las cuales 162 correspondieron a artículos en publicaciones periódicas y 50 a libros y capítulos de libros. Las especies más estudiadas 
fueron Peromyscus yucatanicus, Heteromys gaumeri y Ototylomys phyllotis, en cambio, las que prácticamente carecieron de información fueron Tylomys nudicaudus, Reithrodontomys spectabilis y Heteromys desmarestianus. El estado de Yucatán ha sido el más explorado con el mayor número de localidades y registros de pequeños roedores, seguido de Quintana Roo y finalmente Campeche. Los temas más abordados fueron distribución, taxonomía, filogenia, enfermedades y parasitismo. Se detectaron vacíos de información para la mayoría de las especies en las diferentes temáticas, tales como alimentación, reproducción, anatomía y morfología, por lo que es importante realizar estudios en estos temas que amplíen el conocimiento de los pequeños roedores de la Península de Yucatán. Estas investigaciones podrían aportar información para proponer cambios sobre su estado de protección en las normas mexicanas e internacionales, o con respecto a la conservación de sus hábitats específicos.

Key words: Campeche; diversity; Mexico; Quintana Roo; Rodentia.

๑ 2016 Asociación Mexicana de Mastozoología, www.mastozoologiamexicana.org

\section{Introducción}

La mastofauna mexicana comprende un grupo diverso de vertebrados, con 525 especies que representan el $11 \%$ del total de los mamíferos a nivel mundial, como consecuencia principalmente de una compleja topografía y de la influencia que ejercen las regiones biogeográficas Neártica y Neotropical (Ceballosetal.2005; García-Marmolejoetal.2008). Esta riqueza deespecies ha estimulado la formación de numerosos científicos dedicados al estudio de los mamíferos terrestres en México, generando información numerosa y variada (Guevara-Chumacero et al. 2001; Sosa-Escalante et al. 2013). Como resultado, ha incrementado el número de especies registradas en el país que se debe no sólo a los nuevos descubrimientos, sino también al uso de nuevas técnicas de investigación que incluyen la genética y la ecología molecular (Vázquez-Domínguez et al. 2009; Ramírez-Pulido et al. 2014). De la misma forma, el desarrollo tecnológico de dispositivos para muestreos y recolectas (e.g. trampas cámara, detectores ultrasónicos, radio transmisores con rastreo satelital; $\underline{\text { O'Connell }}$ et al. 2011; Díaz-Pulido y Payán 2012; Boonman et al. 2013), han ayudado a comprender de mejor manera los cambios en la nomenclatura y las relaciones taxonómicas, patrones de distribución y uso de hábitat de especies elusivas. A escala regional, el número de especies registradas para cada entidad federativa cambia de manera frecuente, ya sea por la publicación de nuevos registro qs, o por cambios taxonómicos o de nomenclatura, lo que demuestra la importancia de actualizar periódicamente las compilaciones en existencia (Sosa-Escalante et al. 2013).

Los estudios realizados en pequeños mamíferos como los roedores, han cobrado gran interés ya que proporcionan con cierta facilidad información acerca de sus poblaciones (Fleming 1974; Kelly y Caro 2003). En México, el Orden Rodentia está representado por ocho familias, que incluyen 233 especies de las cuales 180 corresponden a los pequeños roedores (Ramírez-Pulido et al. 2014). Estos pequeños mamíferos resaltan como componentes clave en los procesos de sucesión y regeneración de las selvas tropicales, jugando un papel fundamental como agentes depredadores (DeMattia et al. 2004) y dispersores de semillas (Chung y Corlett 2006; Wells et al. 2009), e incluso como polinizadores (Kleizen et al. 2008), consumidores de invertebrados (Hernández-Betancourt et al. 2005) y vertebrados pequeños (Chung y Corlett 2006), además de ser presas de otras especies (Martínez-Vázquez et al. 2010).

En la Península de Yucatán, provincia biogeográfica separada de las adyacentes por una flora y fauna particular en la que resaltan numerosas especies endémicas (Morrone et al. 2002; VázquezDomínguez y Arita 2010), se distribuyen tres familias de pequeños roedores, Heteromyidae, Cricetidae y Muridae con un total de 15 especies, de las cuales ocho son endémicas de Mesoamérica (53.3\%) y una de México (6.6 \%; Hernández-Betancourt et al. 2010; Sosa-Escalante et al. 2013). En esta península se han desarrollado estudios que han incrementado el conocimiento de las 
comunidades de pequeños roedores en diferentes puntos geográficos (Hernández-Betancourt et al. 2010; Guzmán-Soriano et al. 2013; Sosa-Escalante et al. 2014; Vargas et al. 2014). El presente artículo realiza un análisis sobre la bibliografía publicada desde el año 1901 al 2015, sobre los pequeños roedores de los estados de Campeche, Yucatán y Quintana Roo que conforman la Península de Yucatán en México. Asimismo, se presenta una revisión de la información contenida en la literatura para identificar la temática y cobertura geográfica de los registros de las especies, con el propósito de analizar las perspectivas en el estudio de pequeños roedores y contribuir a los esfuerzos encaminados a su protección y conservación.

\section{Materiales y métodos}

Selección yorganización de la información. Se realizó una búsqueda documental mediante la consulta de literatura existente tanto en medios impresos como electrónicos, partiendo del estudio de Merriam (1901- primer trabajo que reporta alguna de las especies de pequeños roedores para la Península de Yucatán), hasta lo publicado en el mes de julio del año 2015 para los estados de Campeche, Yucatán y Quintana Roo, en torno a las 15 especies de pequeños roedores que se distribuyen en la península. Se tomaron en cuenta aquellos estudios que incluyeran descripciones de nuevas especies, registros y revisiones sobre los pequeños roedores de la Península de Yucatán. Se revisaron exhaustivamente bases de datos de diversas bibliotecas electrónicas (Scielo, BioOne, Biodiversity Heritage Library, Catálogo-UNAM, Web of Science) para la recopilación y revisión detallada de los trabajos en revistas periódicas, de la serie Mammalian Species, libros y capítulos de libros. Se excluyeron resúmenes y memorias de congresos, simposios, reuniones, informes internos y tesis.

Toda la información recabada fue organizada en una base de datos conformada por los campos de autor, año, título, revista, país de origen (nacional o extranjero, que fue definido por el lugar de origen del primer autor), cita bibliográfica, localidad (incluyó combinaciones entre las entidades federativas analizadas y si los trabajos incluyeron localidades de los tres estados entonces se registró como para toda la península), tema principal, especie y observaciones. Para facilitar el manejo de la información, cada trabajo publicado fue etiquetado en un tema principal acorde a su contenido (Guevara-Chumacero et al. 2001). Adicionalmente, se elaboró una base de datos con la información sobre las localidades de muestreo de cada una de las especies de roedores recabada de las publicaciones de interés con los campos de: cita bibliográfica, año, familia, especie, estado, localidad, región, latitud, longitud y observaciones.

Análisis de la información. Por medio de representaciones gráficas se analizó y comparó la información organizada en las respectivas bases de datos. Se elaboró una curva de acumulación de especies por década para cada estado de la Península de Yucatán. Se elaboraron mapas de distribución de los pequeños roedores, a partir de los registros encontrados en las publicaciones recopiladas para los estados de Campeche, Quintana Roo y Yucatán, haciendo uso del programa ArcMap ver. 9.3. Con todos estos análisis en conjunto se pudieron contrastar los esfuerzos que han existido en la generación de publicaciones científicas por décadas, por estados, entre especies y entre temas de investigación.

\section{Resultados}

Análisis de la bibliografía y localidades de muestreo. Se registraron un total de 212 referencias con una o más especies de los pequeños roedores para los estados de la Península de Yucatán, de las cuales $162(76.4 \%)$ corresponden a trabajos en publicaciones periódicas y 50 (23.6\%) pertenecen a libros y capítulos de libros. El número de publicaciones exclusivas para el estado de Yucatán fue de 46 (21.7\%), seguido por Campeche con 35 (16.5\%), y Quintana Roo con el menor número, 23 
(10.9\%). Simultáneamente 78 (36.8\%) referencias abarcaron localidades de los tres estados de la Península de Yucatán. Las publicaciones que incluyeron localidades tanto del estado de Yucatán como de Quintana Roo fueron 13 (6.1\%), para Campeche y Quintana Roo sumaron 9 (4.2 \%) y en conjunto Campeche y Yucatán acumularon 8 (3.8 \%) referencias. Del total de las publicaciones analizadas 123 (58 \%) fueron escritas por autores extranjeros y 89 (42\%) por mexicanos. Para el periodo de 1901-1970 se publicó el $23 \%$ del total de los artículos, mientras que entre 1971-2015 fue el $77 \%$ del total de las publicaciones (Figura 1).

Diversidad de especies, publicaciones y registros por estado. De las 15 especies registradas para la península, $54 \%$ son terrestres, $33 \%$ semi-arborícolas y $13 \%$ arborícolas (Tabla 1). Las especies con más publicaciones y registros fueron Peromyscus yucatanicus, Ototylomys phyllotis y Heteromys gaumeri. En cambio, Tylomys nudicaudus, Reithrodontomys spectabilis y Heteromys desmarestianus han sido las menos registradas y reportadas en publicaciones (Figura 2). En la década de 1950 se confirmó la presencia de dos especies de pequeños roedores para Yucatán, $H$. desmarestianus (Laurie 1953) y T. nudicaudus (Hatt 1953), que representan los últimos registros de pequeños roedores para el estado. A inicios de la década del 2000 se presentó el registro de $H$. desmarestianus en Campeche (Hernández-Huerta et al. 2000) y en Quintana Roo la última confirmación de una especie de pequeño roedor fue Oligoryzomys fulvescens en el año 2009 (Escobedo-Cabrera et al. 2009; Figura 3). Para la década de 1970 ya se tenían registradas a nivel península, las 15 especies de pequeños roedores conocidas hasta el momento.

Análisis de la cobertura geográfica y temas de investigación. Para el periodo de 1901-2015 se cuantificaron en la Península de Yucatán un total de 1,094 registros de pequeños roedores distribuidos en 173 localidades, de los cuales 504 registros (46.1\%) correspondieron a 70 localidades de Yucatán, 309 (28.2 \%) a 62 localidades de Quintana Roo y 281 (25.7\%) a 41 localidades de Campeche. El número de especies registradas por localidad para cada estado fue

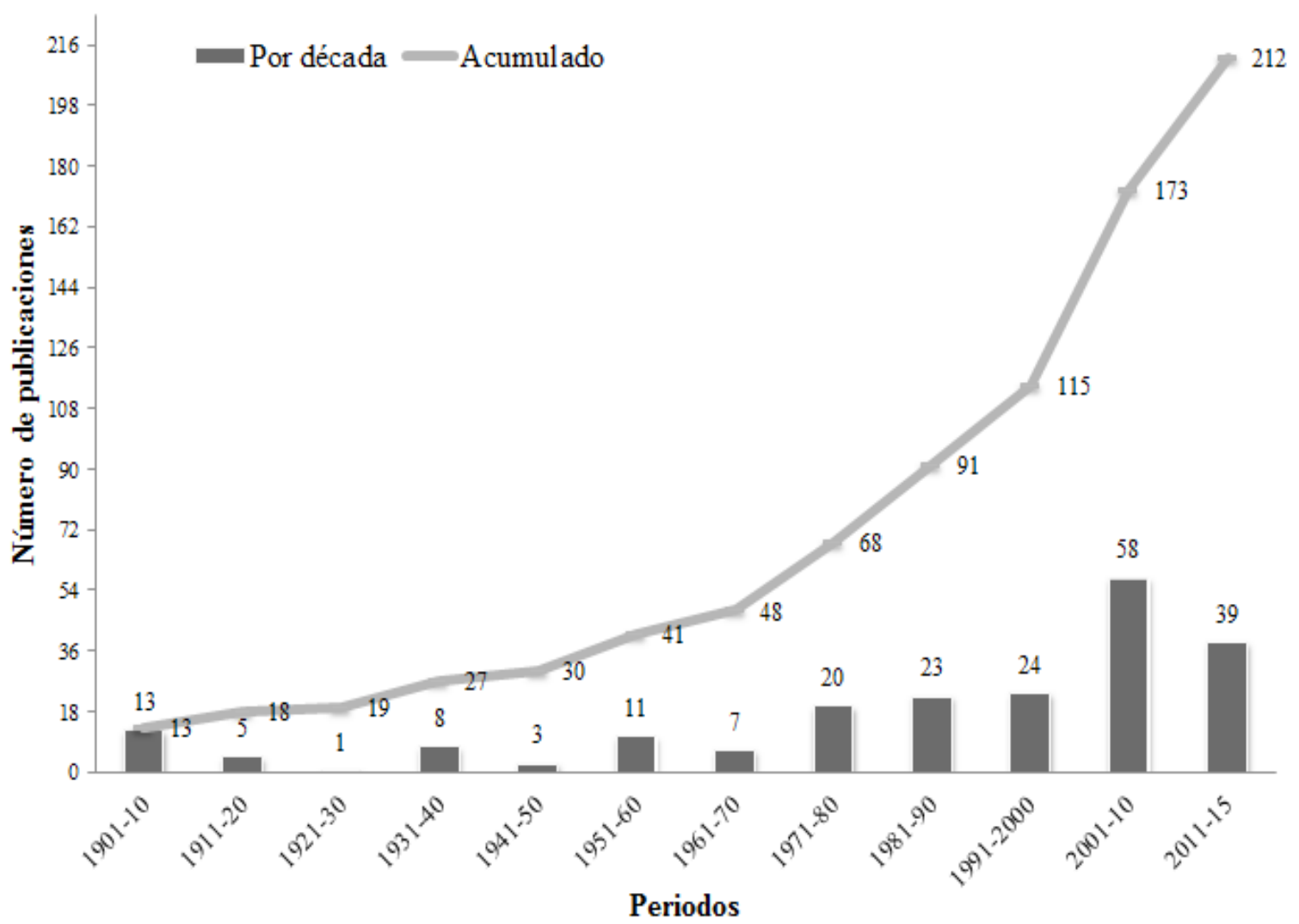

Figura 1. Número de publicaciones sobre los pequeños roedores de la Península de Yucatán por década entre los años 1901 - 2015. 
distinto, Yucatán y Quintana Roo mostraron un mayor número de localidades con una a dos especies registradas, mientras que Yucatán presentó en su mayoría localidades con cuatro a seis o más especies registradas a lo largo de su territorio (Figura 4). La presencia y distribución de los pequeños roedores fue variada a lo largo de la provincia biogeográfica de la península (Figura 5), Yucatán y Quintana Roo son los estados que albergan 14 de las 15 especies registradas hasta el momento, mientras que Campeche presenta 13 especies en su territorio (Tabla 1).

Los temas más abordados en los estudios de pequeños roedores en la Península de Yucatán fueron los de distribución, enfermedades, parasitismo, taxonomía y filogenia con más de 600 trabajos en conjunto, en tanto que los menos estudiados han sido los de anatomía y morfología, reproducción y alimentación (Tablas 1, 2). Las especies $H$. gaumeri, O. phyllotis, Peromyscus leucopus, P. yucatanicus y Sigmodon toltecus contaron con al menos una publicación para la mayoría de los temas, mientras que las especies $H$. desmarestianus, $R$. spectabilis y T. nudicaudus han sido de las menos estudiadas.

En el estado de Yucatán se ha desarrollado la única investigación reportada hasta el momento en referencia a alimentación (Tabla 2), realizada en la especie H. gaumeri. Para el tema de anatomía y morfología, los tres estados de la Península de Yucatán han reportado estudios para las especies $H$. gaumeri, Handleyomys rostratus, P. leucopus, $P$. yucatanicus, Reithrodontomys gracilis y $S$. toltecus. Las tres entidades contaron con trabajos sobre reproducción, correspondientes a las especies Otonyctomys hatti, $O$. phyllotis, P. leucopus y P. yucatanicus (Tabla 2).

Tabla 1. Número de publicaciones por tema principal para cada especie de pequeños roedores durante el periodo 1901 - 2015 en la Península de Yucatán. Las especies registradas con PY se encuentran en los tres estados. El arreglo taxonómico es de acuerdo a Carleton y Musser (2005) y Weksler (2006).

\begin{tabular}{|c|c|c|c|c|c|c|c|c|c|c|c|c|}
\hline Especie & En & A & AM & C & Cn & D & $\mathbf{E}$ & EP & M & $\mathbf{R}$ & TF & TOTAL \\
\hline \multicolumn{13}{|l|}{ Familia Heteromyidae } \\
\hline 'Heteromys desmarestianus & PY & & & & 3 & 18 & & 3 & & & 4 & 28 \\
\hline${ }^{1}$ Heteromys gaumeri & PY & 1 & 1 & 3 & 5 & 39 & 10 & 19 & 4 & & 17 & 99 \\
\hline \multicolumn{13}{|l|}{ Familia Cricetidae } \\
\hline${ }^{2}$ Peromyscus leucopus & PY & & 3 & 4 & 3 & 32 & 2 & 7 & 2 & 1 & 15 & 69 \\
\hline${ }^{2}$ Peromyscus yucatanicus & PY & & 3 & 5 & 4 & 43 & 7 & 24 & 3 & 3 & 15 & 107 \\
\hline${ }^{2}$ Reithrodontomys gracilis & PY & & 1 & 2 & 2 & 34 & 5 & 5 & & & 16 & 65 \\
\hline${ }^{2}$ Reithrodontomys spectabilis & QR & & & 1 & 1 & 12 & 2 & & & & 9 & 25 \\
\hline${ }^{1}$ Handleyomys rostratus & PY & & 1 & 4 & 1 & 23 & 1 & 10 & 1 & & 6 & 47 \\
\hline 'Oligoryzomys fulvescens & PY & & & 2 & 2 & 23 & & 2 & 1 & & 3 & 33 \\
\hline 'Oryzomys couesi & PY & & & 4 & 2 & 34 & 2 & 5 & 3 & & 13 & 63 \\
\hline 'Sigmodon toltecus & PY & & 1 & 4 & 2 & 27 & 3 & 18 & 4 & & 8 & 67 \\
\hline${ }^{3}$ Otonyctomys hatti & PY & & & 1 & & 31 & & 1 & 1 & 1 & 5 & 40 \\
\hline${ }^{2}$ Ototylomys phyllotis & PY & & & 4 & 3 & 37 & 5 & 18 & 5 & 3 & 16 & 91 \\
\hline${ }^{3} T y l o m y s$ nudicaudus & $Y$ & & & & & 10 & & 1 & 1 & & & 12 \\
\hline \multicolumn{13}{|l|}{ Familia Muridae } \\
\hline${ }^{1}$ Mus musculus & PY & & & & 1 & 12 & 5 & 8 & 1 & & 3 & 30 \\
\hline \multirow[t]{2}{*}{${ }^{1}$ Rattus rattus } & PY & & & 1 & 1 & 13 & 1 & 13 & 1 & & 2 & 32 \\
\hline & & 1 & 10 & 35 & 30 & 388 & 43 & 134 & 27 & 8 & 132 & 808 \\
\hline
\end{tabular}

Entidad (En), Yucatán (Y), Quintana Roo (QR), Península de Yucatán (PY), Alimentación (A), Anatomía y Morfología $(A M)$, Colecciones (C), Conservación (Cn), Distribución (D), Ecología (E), Enfermedades y Parasitismo (EP), Misceláneos (M), Reproducción (R), Taxonomía y Filogenia (TF), terrestre ('), semi-arborícola (2), arborícola ( $\left.{ }^{3}\right)$. 


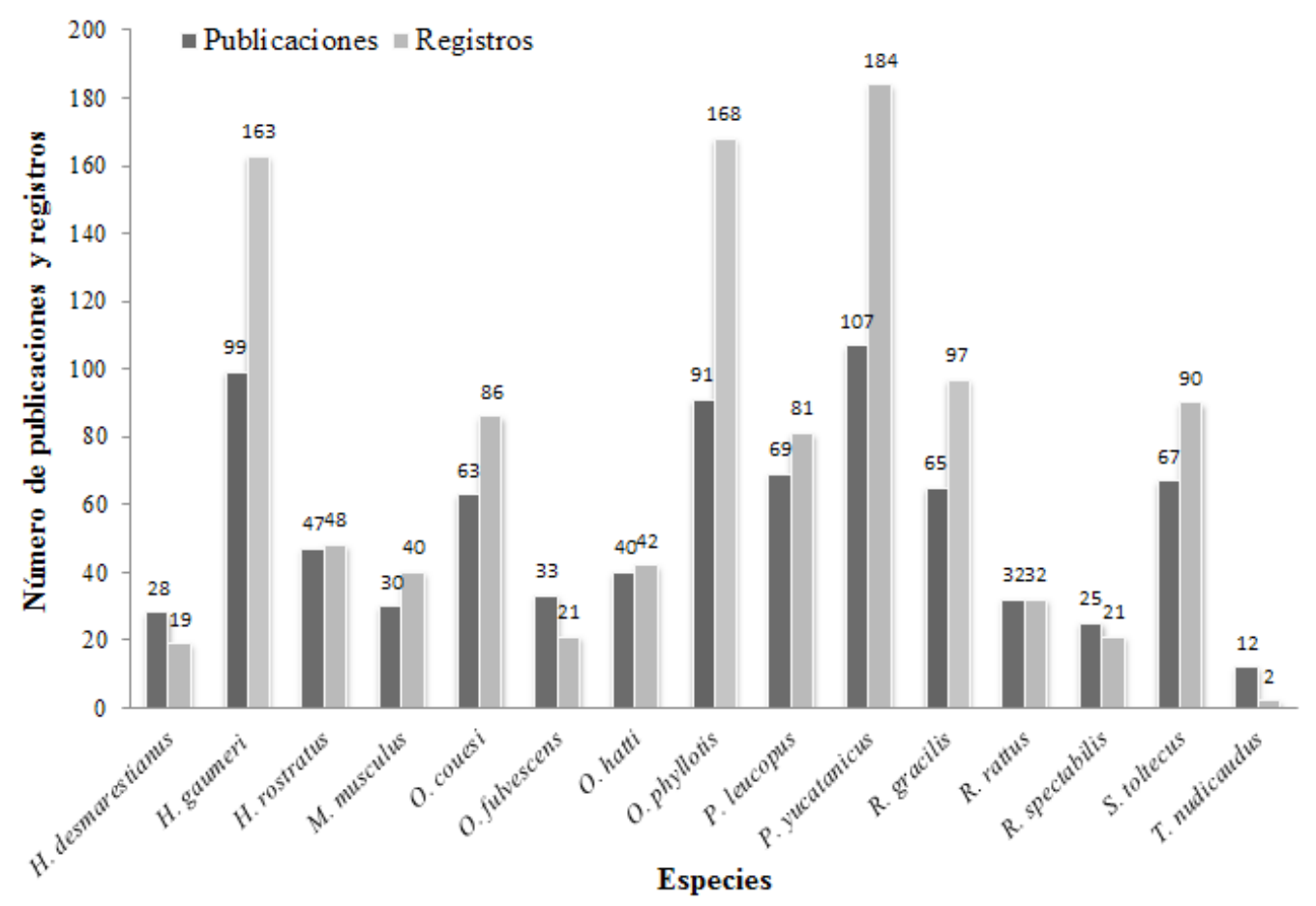

Figura 2. Número de publicaciones y registros por especie en la Península de Yucatán durante el periodo 1901 - 2015.

\section{Discusión}

El estudio de los mamíferos terrestres, así como el de los pequeños roedores en la Península de Yucatán han adquirido un auge notable en México en las últimas décadas. En un principio existió una intensa participación de investigadores extranjeros, pero con el paso del tiempo el número de mastozoólogos mexicanos creció de manera considerable, como un esfuerzo renovado por conocer y estudiar la riqueza biológica en la Península de Yucatán. Este mismo patrón ha sido reportado en otras investigaciones sobre mamíferos de México (Guevara-Chumacero et al. 2001; SánchezCordero y Medellín 2005; Lorenzo et al. 2006) y de manera particular para la Península de Yucatán (Sosa-Escalante et al. 2013, 2014; Vargas et al. 2014).

En la Península de Yucatán se distribuyen el $8 \%$ de los roedores mexicanos (RamírezPulido et al. 2014), de los cuales 11 especies pertenecen a la familia Cricetidae (SosaEscalante et al. 2013), dos a la familia Heteromyidae y dos especies no nativas a la familia Muridae (Guzmán-Soriano et al. 2013; Sosa-Escalante et al. 2014). Esta riqueza regional de especies es resultado posiblemente de las barreras que surgieron de los procesos volcánicos ocurridos en la región norte de Chiapas, que pudieron influir en la migración de estos mamíferos hacia la Península de Yucatán (Espinoza et al. 2006). Conjuntamente, se deben considerar también los cambios climáticos graduales que se presentaron durante el Pleistoceno y que se sabe, contribuyeron a la especiación de los roedores en la región sur de México (Espinoza et al. 2006).

La Península de Yucatán se encuentra por debajo de los 200 metros de altitud (Morrone et al. 2002), con características particulares de tipo de suelo (cárstico) y de flora (Espinoza et al. 2006), producto de su historia geológica y evolutiva que difiere de la del resto del país (Escalante et al. 2007; García-Marmolejo et al. 2008; Vázquez-Domínguez y Arita 2010). Como consecuencia, se pueden hallar en su 


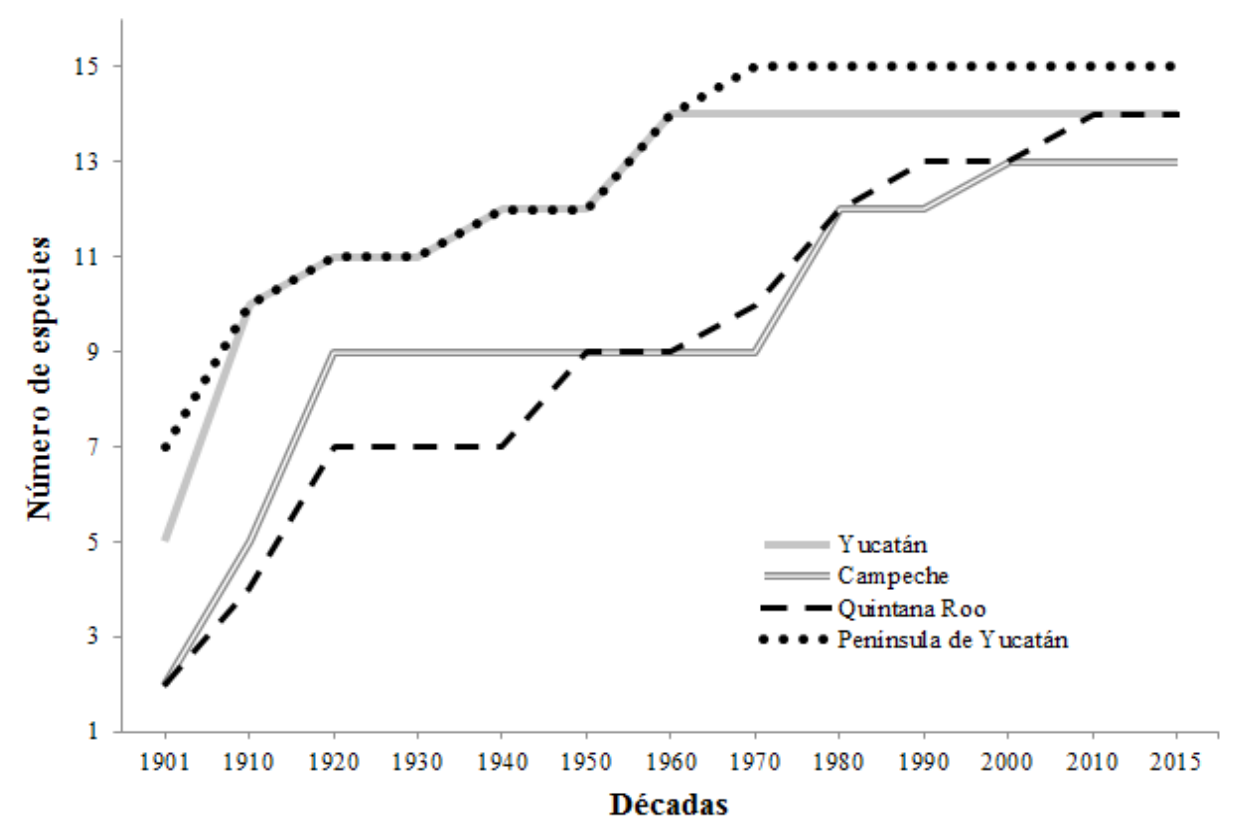

Figura 3. Curva de acumulación de especies de pequeños roedores por década registradas para cada estado de la Península de Yucatán.

territorio especies de pequeños roedores que se distribuyen tanto en la región Neártica como Neotropical y otras endémicas, a pesar de ser considerada como una zona menos diversa que otras regiones del centro y occidente de México (Arita 1997; Vázquez-Domínguez y Arita 2010). Las especies P. leucopus, S. toltecus y Oryzomys couesi son especies que se distribuyen tanto en la región Neártica como Neotropical, mientras que los roedores $O$. hatti, P. yucatanicus y $H$. gaumeri son exclusivos de la provincia biogeográfica de la Península de Yucatán (IUCN 2015). La única especie endémica para México es $R$. spectabilis que se distribuye en la Isla de Cozumel (Knox 1982), el resto de las especies son de distribución Neotropical, con excepción de las introducidas (Rattus rattus y Mus musculus; IUCN 2015).

Cobertura geográfica y temática del estudio de las especies de pequeños roedores. La distribución de los registros por especie mostró un sesgo hacia lugares carismáticos o de importancia arqueológica (i. e. Chichen Itzá, Loltún, Uxmal), así como aquellos sitios de fácil acceso (Sánchez-Cordero y Medellín 2005; Sosa-Escalante et al. 2013), por lo que existen zonas bien definidas en las cuales el registro de pequeños roedores es aún insuficiente. En el estado de Campeche, la franja costera oeste, la parte norte, centro y a lo largo de toda su inmediación fronteriza son zonas con escasa información y con pocas especies de pequeños roedores reportadas. El estado de Quintana Roo, particularmente en la zona central y sus límites territoriales con Campeche, prácticamente no se ha explorado, y a pesar que diversos sitios de la franja costera de Quintana Roo han sido estudiados, aún continúan siendo escasos los registros de especies de roedores. El estado de Yucatán ha sido aparentemente el más explorado; sin embargo, algunos sitios de la franja costera e inmediaciones fronterizas con Campeche y Quintana Roo no han sido estudiados. Esto concuerda con lo encontrado por Sosa-Escalante et al. (2013) en su estudio sobre los mamíferos terrestres de la Península de Yucatán.

De forma general, a lo largo de toda la Península de Yucatán se pueden encontrar las 15 especies de pequeños roedores registradas para la provincia, pero cabe mencionar que algunas de éstas se encuentran muy bien representadas en ciertos 
estados. Por ejemplo, las especies $R$. gracilis, O. couesi, P. leucopus, P. yucatanicus, H. gaumeri y O. phyllotis son las que presentaron mayores registros en Campeche y Yucatán. En contraste, $O$. hatti, H. desmarestianus y $O$. fulvescens tuvieron bajo número de registros.

Si bien se han llevado a cabo numerosos trabajos en toda la Península de Yucatán, en referencia a los listados de distribución de las 15 especies de pequeños roedores, aún son pocos los estudios poblacionales realizados a mediano y largo plazo. Entre éstos destacan la investigación de Hernández-Betancourt et al. (2008b) sobre la dinámica poblacional de la rata arborícola O. phyllotis y el de Cimé-Pool et al. (2002), en el que analizaron el área de actividad de H. gaumeri, ambos estudios efectuados en una selva mediana subcaducifolia del sur de Yucatán. Asimismo destaca el trabajo de Escobedo-Cabrera et al. (2009) realizado desde 1996 hasta el 2005, en el que enlistan los registros de las especies de mamíferos terrestres recabados en áreas fronterizas de los ambientes aledaños al Santuario del Manatí, la ribera del río Hondo y sus áreas de influencia para el estado de Quintana Roo. En este trabajo se reportan las capturas de H. rostratus, O. couesi, O. phyllotis, P. yucatanicus, S. toltecus y H. gaumeri, que serían los registros más sureños para estas especies, sin representación en los mapas aquí presentados, ya que en la publicación no se especifica la localidad exacta de cada registro. Lo anterior resalta la importancia de efectuar una descripción de la ubicación geográfica cuando se realizan investigaciones en áreas de grandes extensiones, sobre todo en aquellas especies cuya distribución se encuentra restringida o poco conocida para la Península de Yucatán.

Los roedores de hábitos arborícolas han sido poco estudiados, ya que la mayoría de los trabajos sobre pequeños roedores se han desarrollado colocando las trampas a nivel de piso, lo que restringe el muestreo de aquellas especies que suelen usar el estrato arbóreo. Este sesgo en el muestreo puede limitar la obtención de nuevos registros para ciertas especies, que exista poca información sobre la biología dealgunos roedores y tener representaciones erróneas acerca de sus densidades poblacionales (Malcolm 1991); Vieira 1998). Un caso particular es la especie arborícola T. nudicaudus que ha sido registrada para la Península de Yucatán únicamente por medio de registros fósiles (Hatt 1953). No obstante, algunos autores reconocen actualmente la posible distribución de T. nudicaudus al interior de la península (Lorenzo et al. 2008;

Tabla 2. Número de publicaciones por tema principal para los estados de la Península de Yucatán durante el periodo $1901-2015$.

\begin{tabular}{lrrrrrrrr}
\hline \multicolumn{1}{c}{ Tema principal } & C & Y & Q & PY & CY & CQ & YQ & TOTAL \\
\hline Alimentación & & 1 & & & & & & 1 \\
Anatomía y Morfología & & 1 & & 2 & 3 & 4 & & 10 \\
Colecciones & & 6 & & 27 & & 2 & 35 \\
Conservación & 7 & 7 & 12 & 4 & & & & 30 \\
Distribución & 35 & 62 & 25 & 236 & 2 & & 28 & 388 \\
Ecología & 3 & 28 & 3 & 7 & & 2 & & 43 \\
Enfermedades y Parasitismo & 36 & 46 & 1 & 47 & 2 & & 2 & 134 \\
Misceláneos & & 22 & & & & & 5 & 27 \\
Reproducción & 5 & & & & 2 & & 1 & 8 \\
Taxonomía y Filogenia & 10 & 16 & 16 & 61 & 3 & 11 & 15 & 132 \\
TOTAL & 96 & 189 & 57 & 384 & 12 & 19 & 51 & 808 \\
\hline
\end{tabular}

Campeche (C), Yucatán (Y), Quintana Roo (Q), Península de Yucatán (PY), Campeche-Yucatán (CY), CampecheQuintana Roo (CQ), Yucatán-Quintana Roo (YQ). 


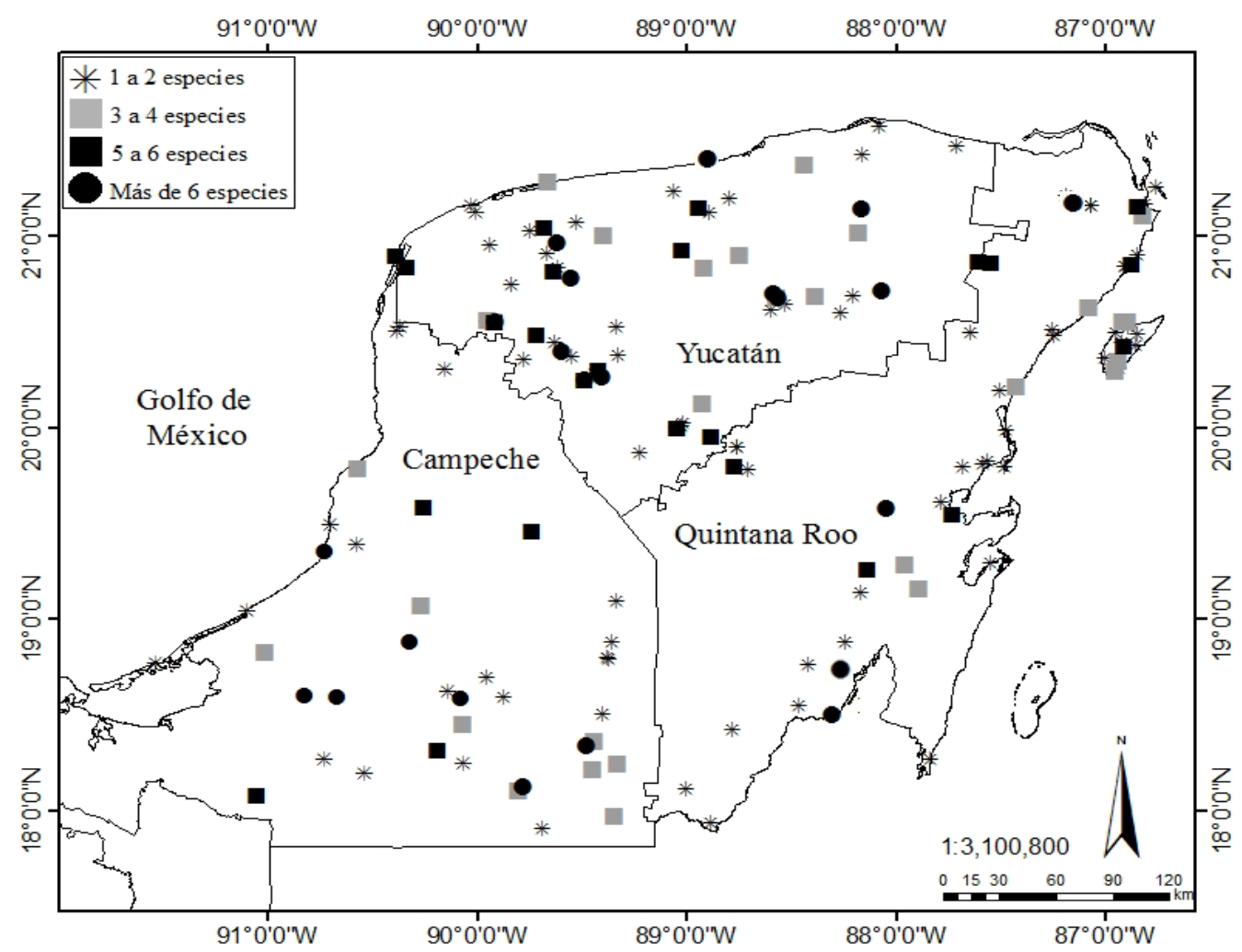

Figura 4. Ubicación geográfica y número de especies registradas por localidad para la Península de Yucatán durante el periodo 1901-2015.

Sosa-Escalante et al. 2014), y su interacción ecológica potencial con otras especies de pequeños roedores que son reservorios de enfermedades, como leishmaniasis (González-Salazar y Stephens 2012).

Otra especie reportada para la Península de Yucatán es el ratón vespertino yucateco O. hatti,cuyos hábitos son también principalmente arborícolas (Hernández-Betancourt et al.2010) y el conocimiento de su biología y ecología han sido muy escasos. En una revisión sobre este roedor, se cuantificaron únicamente 18 especímenes depositados en colecciones científicas y capturados hasta el 2010 provenientes de diferentes localidades de la península, Guatemala y Belice (MacSwiney et al. 2009; Panti-May et al. 2015a). Ha sido de las pocas especies peninsulares en las que se ha efectuado una investigación descriptiva con respecto a su reproducción en cautiverio (Panti-May et al. 2015a). Esto resalta la escasez de trabajos realizados para los pequeños roedores de hábitos arborícolas y semi-arborícolas (Woodman et al. 1995) que se distribuyen en la Península de Yucatán, donde las investigaciones se han enfocado a los inventarios.

La producción científica abordó principalmente la temática de distribución y ecología y en menor número la aplicación de metodologías y enfoques modernos como la taxonomía y filogenia, enfermedades y parasitismo. Sin embargo, las temáticas de alimentación, reproducción, anatomía y morfología, han sido abordadas con pocos trabajos o están ausentes en alguno de los tres estados de la península. Las especies que han sido más estudiadas en la mayoría de los temas fueron P.yucatanicus, H. gaumeri y O. phyllotis, lo que puede ser resultado de su elevada abundancia donde han sido registradas, lo que ha permitido un mayor éxito en investigaciones a largo plazo sobre su biología reproductiva y aspectos ecológicos (Hernández-Betancourt et al. 2003; Hernández-Betancourt et al. 2005; Hernández-Betancourt et al. 2008a; b). Por ejemplo, el único estudio que reporta el posible consumo de caracoles por la especie 
H. gaumeri, resultó de una investigación sobre la ecología de este roedor (HernándezBetancourt et al.2005), mismo que se incluyó dentro del tema de alimentación (dieta), por la relevancia de sus observaciones.

Otras especies han sido objeto de estudios sobre enfermedades transmisibles al ser humano, como por ejemplo $P$. yucatanicus, que se le ha considerado una de las principales especies reservorio de Leishmania sp. en la Península de Yucatán (LoríaCervera et al. 2013). En investigaciones experimentales para comprender la ecología de la Leishmania, este mismo roedor ha sido usado como modelo con la finalidad de generar información sobre los ciclos de transmisión y para contribuir al control y erradicación de esta enfermedad, que afecta a seres humanos y animales domésticos (Sosa-Bibiano et al. 2012; Loría-Cervera y Andrade-Narváez 2014). Otros autores han revisado el papel de varias especies de pequeños roedores de la península como reservorios y transmisores de diversas enfermedades como hantavirus, rickettsiosis, ehrlichiosis, leishmaniasis y Chagas (Reyes-Novelo et al. 2011). En especies introducidas como M. musculus y $R$. rattus, los estudios relacionados a temas de distribución, enfermedades y parasitismo han cobrado importancia ya que son roedores con estrecha asociación con las actividades humanas, que interactúan con especies locales y son transmisoras de diversas enfermedades en la región (VadoSolís et al. 2002; Panti-May et al. 2012; Torres-Castro et al. 2014; Panti-May et al. 2015b). Sin embargo, se requiere un mayor número de estudios sobre esta temática, particularmente en el estado de Quintana Roo, donde hasta ahora el número de trabajos ha sido muy bajo.

Estado de conservación de los pequeños roedores en la Península de Yucatán. Los ecosistemas que componen la Península de Yucatán han sido constantemente perturbados por la acción humana durante varios miles de años (Turner y Butzer 1992). Como resultado, la vegetación actual es un mosaico de sucesión de selvas, por efecto de al menos 3,000 años de manipulación humana, incluyendo la agricultura, la explotación forestal, la urbanización y disturbios naturales como los incendios y huracanes (Schultz 2003). En parte, es posible que lo anterior haya propiciado que varias especies estén catalogadas en alguna categoría de riesgo. Éste es el caso para $O$. couesi, cuya subespecie $O$. couesi cozumelae está catalogada como amenazada, situación similar a lo que ocurre con $P$. leucopus cozumelae y $R$. gracilis insularis (SEMARNAT 2010). El ratón vespertino O. hatti también está considerado como amenazado ante la pérdida de su hábitat (Sosa-Escalante et al. 2013) y R. spectabilis como un especie amenazada (SEMARNAT 2010) y por la IUCN como en peligro crítico (IUCN 2015). Es relevante mencionar que estas especies y subespecies, aún no cuentan con estudios que permitan conocer el estado actual de sus poblaciones silvestres.

Actualmente también existe un serio problema con las especies que han sido introducidas, particularmente en la Isla Cozumel, entre las que se encuentran roedores exóticos, gatos y perros ferales, además de la liberación de boas (Boa constrictor) en 1971, que en conjunto están impactando negativamente a los vertebrados de la isla (Martínez-Morales y Cuarón 1999; Harper y Bunbury 2015). Esto aumenta el riesgo de peligro para las especies amenazadas en la zona como los roedores $O$. couesi cozumelae (Vega et al. 2007), R. spectabilis y P. leucopus cozumelae cuyas poblaciones hoy día son desconocidas en términos de su hábitos alimenticios, anatomía, morfología y aspectos reproductivos para la península.

El hecho de que algunos de los pequeños roedores de la Península de Yucatán estén bajo alguna categoría de riesgo, debiera motivar la generación de conocimiento 
con el fin de proponer estrategias efectivas para su conservación. Cabe enfatizar que tanto la biología de las especies como su comportamiento, influyen en su captura y en el número reducido de registros. Si bien es posible que algunas especies de pequeños roedores como $R$. spectabilis, $H$. desmarestianus, P. leucopus, O. hatti y T. nudicaudus se encuentren a lo largo del territorio peninsular (muchos han sido reportados por avistamientos ocasionales), hasta ahora han existido dificultades para estudiar estas especies (e. g. baja densidad poblacional, limitaciones en el muestreo).

Perspectivas en el estudio de los pequeños roedores en la Península de Yucatán. A través de la información acumulada se reconoce a los pequeños roedores como un grupo que se distingue por las funciones imprescindibles en los ecosistemas y por el grado de riesgo de extinción de algunas especies (Ceballos 2005). Además, numerosas especies han sido consideradas indicadores ecológicos (Cimé-Pool et al. 2007; Cimé-Pool et al. 2010; Whitehead et al. 2014), lo que enfatiza la necesidad de continuar realizando estudios sobre su dinámica poblacional, ya que de esta manera se aportaría información, por ejemplo, sobre las modificaciones del hábitat en la península.

A pesar de la información incluida en las 212 publicaciones sobre los pequeños roedores en la Península de Yucatán, continúa un desconocimiento sobre aspectos básicos de biología de las especies de la región, ya que la mayoría de estudios han

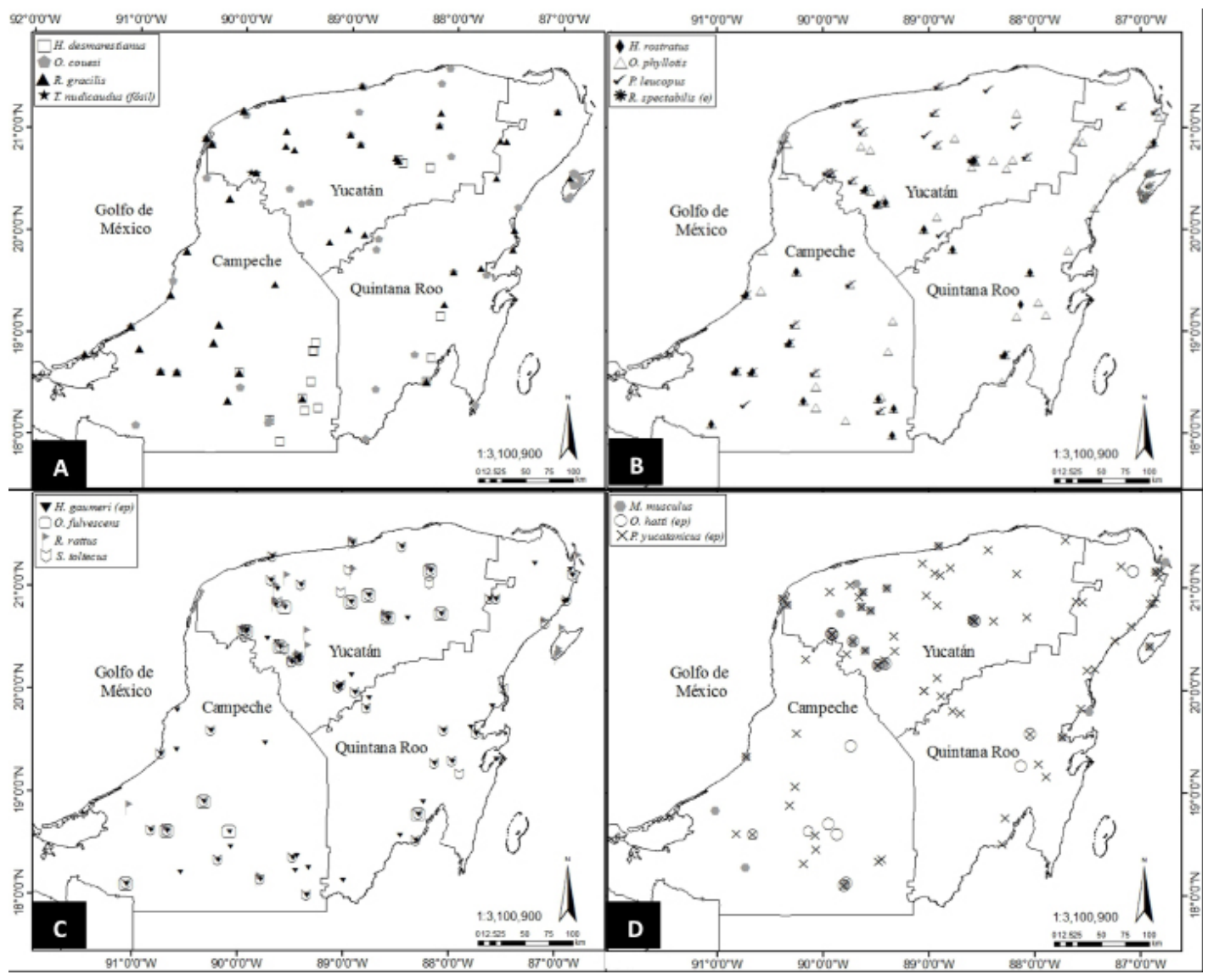

Figura 5. Ubicación geográfica de las 15 especies de pequeños roedores registrados en la Península de Yucatán durante el periodo 1901-2015. Mapa A: Heteromys desmarestianus, Oryzomys couesi, Reithrodontomys gracilis y Tylomys nudicaudus. Mapa B: Handleyomys rostratus, Ototylomys phyllotis, Peromyscus leucopus y Reithrodontomys spectabilis. Mapa C: Heteromys gaumeri, Oligoryzomys fulvescens, Rattus rattus y Sigmodon toltecus. Mapa D: Mus musculus, Otonyctomys hatti y Peromyscus yucatanicus. e = endémica de México, ep = endémica de la Península de Yucatán. 
sido realizados de forma esporádica y son principalmente inventarios. Es prioritario que se realice investigación con muestreos sistemáticos y a largo plazo y que aborden temáticas de ecología poblacional, alimentación, reproducción, anatomía, morfología y conservación. También es prioritario que se realicen investigaciones en aquellas zonas que se han identificado con vacíos de información. Sólo con datos generados de estas investigaciones se podrán hacer recomendaciones fundamentadas para determinar el estado de prioridad de conservación de las especies de roedores en las normas mexicanas (i. e. NOM-059-SEMARNAT-2010) e internacionales (i. e. Lista Roja de Especies Amenazadas IUCN), o con respecto a la conservación de sus hábitats específicos (e.g. R. spectabilis, P. leucopus cozumelae y $O$. couesi cozumelae restringidas a la Isla Cozumel, $R$. gracilis insularis endémica de Isla del Carmen, O. hatti, H. gaumeri y P. yucatanicus endémicas de la Península de Yucatán), y a su vez, incrementar el conocimiento de los mamíferos de esta provincia biogeográfica.

\section{Agradecimientos}

El primer autor agradece al Consejo Nacional de Ciencia y Tecnología (CONACyT) por el apoyo de la beca 297705 otorgada para realizar estudios de doctorado. Asimismo, agradecemos a C. Alavez por su apoyo en la búsqueda de coordenadas geográficas, que requirieron algunas de localidades de la base de datos empleada para este trabajo. Agradecemos los comentarios de los tres revisores anónimos que permitieron mejoraron la calidad de este manuscrito.

\section{Literatura citada}

ARITA, H. T. 1997. Species composition and morphological structure of the bar fauna of Yucatan, Mexico. Journal of Animal Ecology 66:83-97.

Boonman, A., Y. Bar-ON, N. Cvikel, y Y. Yovel. 2013. It's not black or white- on the range of vision and echolocation in echolocating bats. Frontiers in Physiology 4:1-12.

Carleton, M. D., y G. G. Musser. 2005. Orden Rodentia. Pp. 745-752 en Mammal Species of the World. A taxonomic and geographic reference. Johns Hopkins University Press. Baltimore, EE. UU.

Ceballos, G. 2005. Los mamíferos de México. Pp. 530-821 en Los mamíferos silvestres de México (Ceballos, G., y G. Oliva, eds.). Fondo de Cultura Económica, Comisión Nacional para el Conocimiento y Uso de la Biodiversidad. Ciudad de México, México.

Ceballos, G., J. Arroyo-Cabrales, R. A. Medellín, L. Medrano y G. Oliva. 2005. Diversidad y conservación de los mamíferos de México. Pp. 21-66 en Los mamíferos silvestres de México (Ceballos, G., y G. Oliva, eds.). Fondo de Cultura Económica, Comisión Nacional para el Conocimiento y Uso de la Biodiversidad. Ciudad de México, México.

Cimé-Pool, J. A, S. F. Hernández-Betancourt, y S. Medina. 2002. Área de actividad de Heteromys gaumeri en una selva mediana subcaducifolia de Yucatán. Revista Mexicana de Mastozoología 6:5-18.

Cimé-Pool, J. A, S. F. Hernández-Betancourt, y J. B. Chablé-Santos. 2007. Comunidad de pequeños roedores en dos agroecosistemas de Yucatán, México. Revista Mexicana de Mastozoología 11:57-68.

Cimé-Pool, J. A., S. F. Hernández-Betancourt, R. C. Barrientos, y A. A. Castro-Luna. 2010. Diversidad de pequeños roedores en una selva baja caducifolia espinosa del noreste de Yucatán, México. Therya 1:23-40. 
Chung, K. P. S, y R. T. Corlett. 2006. Rodent diversity in a highly degraded tropical landscape: Hong Kong, South China. Biodiversity and Conservation 15:4521-4532.

Demattia, E. A., L. M. Curran, y B. J. Rathcke. 2004. Effects of small rodents and large mammals on Neotropical seeds. Ecology 85:2161-2170.

Díaz-Pulido, A., y E. Payán. 2012. Manual de fototrampeo: una herramienta de investigación para la conservación de la biodiversidad en Colombia. Instituto de Investigaciones de Recursos Biológicos Alexander von Humboldt y Panthera Colombia. Bogotá, Colombia.

Escobedo-Cabrera, E., M. Chamblé-Jiménez, y C. Pool-Valdez. 2009. Mamíferos terrestres. Pp. 174-183 en El sistema ecológico de la había de Chetumal/Corozal: costa occidente del Mar Caribe (Espinoza-Ávalos, J., G. A. Islebe, y H. A. Hernández, eds.). El Colegio de la Frontera Sur. Chetumal, México.

Escalante, T., V. Sánchez-Cordero, J. J. Morrone y M. Linaje. 2007. Areas of endemism of mexican terrestrial mammals: a case study using species'ecological niche modeling, parsimony analysis of endemicity and goloboff fit. Interciencia 32:151-159.

Espinoza, D., I. Sánchez, M. García, y C. LoRenzo. 2006. Análisis de la distribución de roedores de la familia Muridae en el sur de México. Pp. 47-54 en Genética y mamíferos mexicanos: presente y futuro (Vázquez-Domínguez, E., y D. J. Hafner, eds.). New Mexico Museum of Natural History and Science Bulletin 32. Albuquerque, EE. UU.

Fleming, T. H. 1974. The population ecology of two species of Costa Rica heteromyid rodents. Ecology 55:493-510.

García-Marmolejo, G., T. Escalante, y J. J. Morrone. 2008. Establecimiento de prioridades para la conservación de mamíferos terrestres neotropicales de México. Mastozoología Neotropical 15:41-65.

González-Salazar, C., y C. R. Stephens. 2012. Constructing ecological networks: a tool to infer risk of transmission and dispersal of Leishmaniasis. Zoonoses and Public Health 59:179-193.

Guevara-Chumacero, L. M., R. López-Wilchis, y V. Sánchez-Cordero. 2001. 105 años de investigación mastozoológica en México (1890-1995): una revisión de sus enfoques y tendencias. Acta Zoológica Mexicana 83:35-72.

Guzmán-Soriano, D., O. G. Retana, y J. D. Cú-Vizcarra. 2013. Lista de los mamíferos terrestres del estado de Campeche, México. Acta Zoológica Mexicana 29:105-128.

Harper, G. A., y N. Bunbury. 2015. Invasive rats on tropical islands: Their population biology and impacts on native species. Global Ecology and Conservation 3:607-627.

HatT, R. T. 1953. The mammals. Pp. 45-77 en Faunal and archeological researches in Yucatan caves (Hatt, R. T., H. I. Fisher, D. A. Langerbartel, y G. W. Brainerd, eds.). Cranbrook Institute of Science Bulletin 33. Bloomfield Hills, EE. UU.

Hernández-Huerta, A., V. J. Sosa, J. M. Aranda, y J. Bello. 2000. Records of small mammals in the Calakmul Biosphere Reserve, Yucatán Peninsula. The Southwestern Naturalist 45:340-344.

Hernández-Betancourt, S. F., R. López-Wilchis, J. A. Cimé-Pool, y S. Medina. 2003. Área de actividad, movimiento y organización social de Heteromys gaumeri Allen y Chapman, 1897 (Rodentia: Heteromyidae) en una selva mediana subcaducifolia de Yucatán, México. Acta Zoológica Mexicana 90:77-91.

Hernández-Betancourt, S. F., J. G. Gómez, J. A. Cimé-Pool, S. Medina, y C. M. Euán-Canul. 2005. First report of use of land snails for Heteromys gaumeri (Rodentia:Heteromyidae) in a subdeciduous forest in Yucatán, México. Acta Zoológica Mexicana 21:155-156.

Hernández-Betancourt, S. F., J. A. Cimé-Pool, y S. Medina. 2008a. Ecología poblacional de Heteromys gaumeri en la selva del sur de Yucatán, México. Pp. 427-448 en Avances en 
el estudio de los mamíferos de México Volumen II (Lorenzo, C., E. Espinoza, y J. Ortega, eds.). Asociación Mexicana de Mastozoología A. C., El Colegio de la Frontera Sur. San Cristóbal de Las Casas, México.

Hernández-Betancourt, S. F., J. A. Cimé-Pool, S. Medina, y M. L. González-Villanueva. 2008 b. Fluctuación poblacional de Ototylomys phyllotis Merriam, 1901 (Rodentia: Muridae) en una selva mediana subcaducifolia del sur de Yucatán, México. Acta Zoológica Mexicana 24:161-177.

Hernández-Betancourt, S. F., C. Sélem, J. A. Cimé-Pool, y J. Chablé. 2010. Diversidad de pequeños roedores de la Península de Yucatán. Bioagrociencias 3:28-31.

iUCN (International Union for Conservation of Nature). 2015. The IUCN Red List of Threatened Species 2015-3.

Kelly, M. J., y T. Caro. 2003. Low density of small mammal community at Las Cuevas, Belice. Mammalian Biology 68:372-386.

KLeizen, C., J. Midgley, y S. D. Johnson. 2008. Pollination systems of Colchicum (Colchicaceae) in Southern Africa: evidence for rodent pollination. Annals of Botany 102:747-755.

Knox, J. 1982. Reithrodontomys spectabilis. Mammalian Species 193:1.

LaURIE, E. M. O. 1953. Rodents from British Honduras, Mexico, Trinidad, Haiti and Jamaica collected by Mr. I. T. Sanderson. Annals and Magazine of Natural History, Series 12, 6:382-394.

Lorenzo, C., E. Espinoza, M. Á. Briones, y F. A. Cervantes (eds.). 2006. Colecciones mastozoológicas de México. Instituto de Biología-UNAM y Asociación Mexicana de Mastozoología, A. C. Ciudad de México, México.

Lorenzo, C., E. E. Espinoza, E. J. Naranjo, y J. Bolaños. 2008. Mamíferos terrestres de la frontera sur de México. Pp.147-164 en Avances en el estudio de los mamíferos de México. Publicaciones especiales Vol. II (Lorenzo, C., E. Espinoza, y J. Ortega, eds.). Asociación Mexicana de Mastozoología, A. C. Ciudad de México, México.

Loría-Cervera, E. N., E. Sosa-Bibiano, L. E. Villanueva-Lizama, N. R. Van, S. B. Canto-Lara, J. L. Batún-Cutz, y F. J. Andrade-Narváez. 2013. Nitric oxide production by Peromyscus yucatanicus (Rodentia) infected with Leishmania (Leishmania) mexicana. Memórias do Instituto Oswaldo Cruz 108:172-177.

Loría-Cervera, E. N., y F. J. Andrade-Narváez. 2014. Animal models for the study of leishmaniasis immunology. Revista do Instituto de Medicina Tropical de São Paulo 56:1-11.

MacSwiney, M. C., S. F. Hernández-Betancourt, y R. Ávila-Flores. 2009. Otonyctomys hatti (Rodentia: Cricetidae). Mammalian Species 825:1-5.

Malcolm, J. R. 1991. Comparative abundances of Neotropical small mammals by trap height. Journal of Mammalogy 72:188-192.

Martínez-Morales, M. A., y A. D. Cuarón. 1999. Boa constrictor, an introduced predator threatening the endemic fauna on Cozumel Island, Mexico. Biodiversity and Conservation 8:957-963.

Martínez-Vázquez, J., R. M. González-Monroy, y D. Díaz-Díaz. 2010. Hábitos alimenticios del coyote en el parque nacional Pico de Orizaba. Therya 1:145-154.

MerRiam, C. H. 1901. Six new mammals from Cozumel, Yucatan. Proceedings of the Biological Society of Washington 14:99-104.

Morrone, J. J., D. Espinosa-Organista, y J. Llorente-Bousquets. 2002. Mexican biogeographic provinces: preliminary scheme, general characterizations, and synonymies. Acta Zoológica Mexicana 85:83-108.

O’Connell, A. F., J. D. Nichols, y K. U. Karanth. 2011. Camera Traps in Animal Ecology: Methods and Analyses. Springer Publishing. Tokyo, Japón. 
Panti-May, J. A., S. F. Hernández-Betancourt, H. Ruíz-Piña, y S. Medina-Peralta. 2012. Abundance and population parameters of commensal rodents present in rural households in Yucatan, Mexico. International Biodeterioration and Biodegradation 66:77-81.

Panti-May, J. A., M. C. MacSwiney, S. F. Hernández-Betancourt, y O. A. Valdes-Rodríguez. 2015 a. Reproduction and postnatal development in the Yucatan vesper mouse. Mammalia 79:169-176.

Panti-May, J. A., S. F. Hernández-Betancourt, R. I. Rodríguez-Vivas, y M. R. Robles. 2015b. Infection levels of intestinal helminths in two commensal rodent species from rural households in Yucatan, Mexico. Journal of Helminthology 89:42-48.

Ramírez-Pulido, J., N. González-Ruiz, A. L. Gardner, y J. Arroyo-Cabrales. 2014. List of Recent Land Mammals of Mexico, 2014: Special Publications. Museum of Texas Tech University 63:1-76.

Reyes-Novelo, E., H. Ruiz-Piña, J. Escobedo-Ortegón, I. Rodríguez-Vivas, M. Bolio-González, A. Polanco-Rodríguez, y P. Manrique-Salde. 2011. Situación actual y perspectivas para el estudio de las enfermedades zoonóticas emergentes, reemergentes y olvidadas en la Península de Yucatán, México. Tropical and Subtropical Agroecosystems 14:35-54.

Sánchez-Cordero, V., y R. A. Medellín (eds.). 2005. Contribuciones mastozoológicas en homenaje a Bernardo Villa. Instituto de Biología-UNAM, Instituto de Ecología-UNAM y Comisión Nacional para el Conocimiento y Uso de la Biodiversidad (CONABIO). Ciudad de México, México.

Schultz, G. P. 2003. Structure and Diversity of the Forests at the El Edén Ecological Reserve. Pp. 91-114 en The Lowland Maya Area: Three Millennia at the Human-Wildland Interface (Gómez-Pompa, A., M. F. Allen, S. L. Fedick, y J. J. Jiménez-Osornio, eds.). The Haworth Press. Albuquerque, EE. UU.

Semarnat (Secretaria del Medio Ambiente y Recursos Naturales). 2010. Norma Oficial Mexicana NOM-059-SEMARNAT-2010, Protección ambiental-especies nativas de México de flora y fauna silvestres-Categorías de riesgo y especificaciones para su inclusión, exclusión o cambio. Lista de especies en riesgo. Diario Oficial de la Federación Segunda sección 30 de diciembre de 2010 1-78.

Sosa-Bibiano, E. I., N. R. Van Wynsberghe, S. B. Canto-Lara, y F. J. Andrade-Narvaez. 2012. Preliminary study towards a novel experimental model to study localized cutaneous leishmaniasis caused by Leishmania (Leishmania) mexicana. Revista do Instituto de Medicina Tropical de São Paulo 54:165-169.

Sosa-Escalante, J. E., J. M. Pech-Canché, M. C. MacSwiney, y S. F. Hernández-Betancourt. 2013. Mamíferos terrestres de la Península de Yucatán, México: riqueza, endemismo y riesgo. Revista Mexicana de Biodiversidad 84:1-21.

Sosa-Escalante, J. E., S. F. Hernández-Betancourt, J. M. Pech-Canché, M. C. MacSwiney, y R. Díaz-Gamboa. 2014. Los mamíferos del estado de Yucatán. Revista Mexicana de Mastozoología Nueva época 4:40-59.

Torres-Castro, M. A., E. Gutiérrez-Ruiz, S. F. Hernández-Betancourt, R. Peláez-Sánchez, P. Agudelo-Flórez, L. Guillermo-Cordero, y F. I. Puerto. 2014. First molecular evidence of Leptospira spp. in synanthropic rodents captured in Yucatan, Mexico. Revue de Médecine Vétérinaire 7-8:213-218.

Turner, B. L., Y K. W. Butzer. 1992. The Columbian encounter and land use change. Environment 34:16-44.

Vado-Solís, I., M. F. Cárdenas-Marrufo, B. Jiménez-Delgadillo, A. Alzina-López, H. LaviadaMolina, V. Suarez-Solís y J.E. Zavala-Velázquez. 2002. Clinical-epidemiological study 
of leptospirosis in humans and reservoirs in Yucatan, Mexico. Revista do Instituto de Medicina Tropical de São Paulo 44:335-340.

Vargas, J. A., G. Escalona, D. Guzmán, O. G. Retana, H. Zarza, y G. Ceballos. 2014. Los mamíferos del estado de Campeche. Revista Mexicana de Mastozoología Nueva época 4:60-74.

Vázquez-Domínguez, E., S. Castañeda-Rico, T. Garrido-Garduño, y T. A. Gutiérrez-García. 2009. Avances metodológicos para el estudio conjunto de la información genética, genealógica y geográfica en análisis evolutivos y de distribución. Revista Chilena de Historia Natural 82:277-297.

Vázquez-Domínguez, E., y H. T. ARItA. 2010. The Yucatan peninsula: biogeographical history 65 million years in the making. Ecography 33:212-219.

Vega, R., E. Vázquez-Domínguez, A. Mejía-Puente, y A. D. Cuarón. 2007. Unexpected high levels of genetic variability and the population structure of an island endemic rodent (Oryzomys couesi cozumelae). Biological Conservation 137:210-222.

VieIRA, E. M. 1998. A technique for trapping small mammals in the forest canopy. Mammalia 62:306-310.

Wells, K., R. T. Corlett, M. B. Lakim, E. K. V. Kalko, y M. Pfeiffer. 2009. Seed consumption by small mammals from Borneo. Journal of Tropical Ecology 25:555-558.

WeksLer, M. 2006. Phylogenetic relationships of Oryzomine rodents (Muroidea: Sigmodontinae): separate and combined analyses of morphological and molecular data. Bulletin of the American Museum of Natural History 296:1-48.

Whitehead, T., M. Goosem, y N. Preece. 2014. Use by small mammals of a chronosequence of tropical rainforest revegetation. Wildlife Research 41:233-242.

Woodman, N., N. A. Slade, R. M. Tımm, y C. A. Schmidt. 1995. Mammalian community structure in lowland, tropical Peru, as determined by removal trapping. Zoological Journal of the Linnean Society 113:1-20.

Submited: January 14, 2016

Reviewed: March 6, 2016

Accepted: March 20, 2016

Associated editor: Consuelo Lorenzo 